AIAA Space 2011 Session EXPL-08 life Support and Habitation Systems

Manuscript 1073768

\title{
Long-Duration Human Habitation Beyond Low-Earth Orbit: Why is the Near Future Critical?
}

\author{
Harley A.Thronson ${ }^{1}$ \\ NASA Goddard Space Flight Center, Greenbelt, MD, 20771 \\ Daniel Lester ${ }^{2}$ \\ Department of Astronomy, University of Texas, Austin, $T X, 78712$ \\ and \\ C. A. "Skip" Hatfield" \\ NASA Johnson Space Center, Houston, TX, 77058
}

You can explore beyond LEO with the systems that you have or wait for the systems that you wish you had.

For more than a decade, habitation systems capable of comfortable human occupation and effective operations beyond low-Earth orbit (LEO) for more than a few weeks have been a priority recommendation to NASA. This capability is a lynchpin for human exploration beyond the Earth-Moon system. Here we describe briefly some relevant concepts and discuss justifications in the current political and financial environment for why near-term human habitation systems beyond LEO is an imperative.

\section{Introduction}

$\mathrm{T}$ he past decade, beginning with the 1999/2000 Decade Planning Team (DPT)/NASA Exploration Team (NExT) ${ }^{1}$ human space flight studies for the White House Office of Management and Budget, can fairly be described as a Golden Age of engineering design, strategic planning, technology capability prioritization, and future concepts. On the other hand, cynics have criticized the same period as little more than PowerPoint presentations, unfocused technology investments, and lost opportunities with only limited progress toward a goal of human exploration beyond the immediate vicinity of the Earth.

We disagree with the cynics: the increasingly sophisticated designs, development of investment roadmaps, and critically - experience with the International Space Station have prepared NASA and multiple partners to deploy the next major "stepping stone" for exploration: human habitation systems beyond low-Earth orbit within about a decade. We shall discuss in this paper why such a near-term activity could be a major near-term achievement in human space flight for NASA and its international partners.

We begin with an overview of recent work on designs for habitation systems that may be deployed in cis-lunar space and their operation.

\footnotetext{
Senior Scientist for Advanced Concepts, Science and Exploration Division, NASA Goddard Space Flight Center, Greenbelt, MD, 20771, Member, AlAA

${ }^{2}$ Research Astronomer, Department of Astronomy, University of Texas, Austin, TX, 78712

${ }^{3}$ Manager, International Space Station Program/Project Integration, NASA Johnson Space Center, Houston, TX, 77058 , Senior Member, AIAA
} 


\section{Summaries of Some Current US Concepts for Cis-Lunar Human Habitation}

Long-duration human operations beyond low-Earth orbit (LEO) in cis-lunar space seem the obvious next major capability for human space flight. Although there are three plausible subsequent destinations (near-Earth asteroids, the lunar surface, and Mars), many human space flight architectures emphasize operations in cis-lunar space, including further development on the International Space Station (ISS), as a precursor or test site for missions deeper into space. In the following discussion, we assume that before the longest-duration human space expeditions can take place, it will be necessary to develop the capability to operate comfortably beyond LEO for substantially longer than the 21 -day limit of the current design for the NASA Multi-Purpose Crew Vehicle (MPCV), although not for as long as required for a voyage to Mars and back (i.e., $\sim 18$ months or

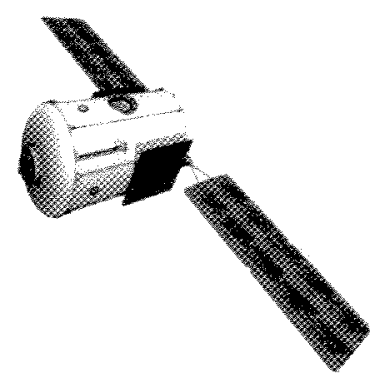

Figure 1: One concept for the the Deep Space Habitat (DSH). In this design, the requirements for the DSH have been satisfied by a metallic cylinder of approximately $8 \mathrm{~m} \times 5 \mathrm{~m}$. For very long voyages, this module will have to include additional systems, such as a propulsion unit. more). That is, exploiting the ISS to the fullest, followed by a habitation system capable of supporting human operations for up to a few months beyond LEO, is an obvious bridge to subsequent, more-challenging human voyages far beyond the Earth-Moon system. In addition to demonstrating those very-long-duration capabilities in a venue that permits easy return to Earth, there are useful tasks that may be carried out by human/robotic systems in these locations. Moreover, several design studies have assessed the value of cis-lunar obits, especially the libration points, as candidate "jumping off" locations for human mission deeper into space.

We emphasize that responsibly limiting the capabilities of the habitation systems briefly summarized here allows major cost and schedule savings, thus significantly advancing human exploration beyond LEO.

\section{A. The Deep Space Habitat (DSH)}

The NASA Human Architecture Team (HAT) developed over the past several months the basic characteristics and requirements of a long-duration habitation system capable of operating in the Earth-Moon system. ${ }^{5}$ However, it is intended eventually for very long voyages, specifically to near-Earth asteroids (NEAs) with durations up to $\sim 1$ year. Thus, the DSH requires capabilities that a purely cis-lunar human habitat does not, at least in the near term. That is, the DSH as currently described has far more capability in some respects than might be necessary for a program intended for near-term deployment. Therefore, some type of "DSH-lite" that is more modest than that described by the HAT could be very attractive for early deployment, as it would seem to be more readily upgraded over time to its full capability than some other habitat concepts.

Figure 1 shows the incarnation of the DSH that uses a rigid cylinder as the basic habitat outer structure, although an inflatable option or mixed rigid/inflatable design may be an equally satisfactory option.

\section{B. The ISS: Existing Hardware and the "Base Camp"}

With the ISS complete and lessons learned from the experience of its management and constructions, some teams have developed concepts that take advantage of unused hardware as building blocks for habitation systems beyond LEO in the near term. ${ }^{6,7}$ The advantages of this path are obvious: core elements of a future human operations site have already been constructed and, to some degree, undergone advanced testing. For example, the available ISS Node STA could be adapted as the central docking structure and the ISS Multi-Purpose Logistics Module (MPLM), of which two are available, could serve as living and operations quarters for the astronauts. In the existing designs the Shuttle

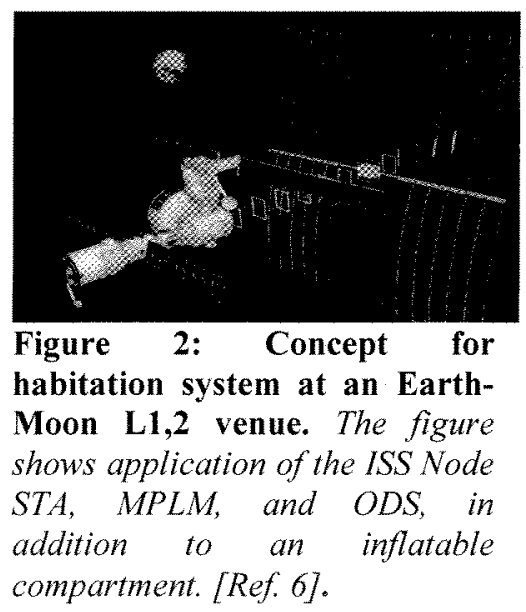

\footnotetext{
* In this paper, we will usually emphasize the Earth-Moon L1 or L2 locations as attractive venues for a sustained human presence beyond LEO, as discussed by many authors elsewhere (See, e.g., Refs. 1, 2 and references therein). For historical interest, probably the first popular reference to operations at these sites was Arthur C. Clarke's $A$ Fall of Moon Dust (1961), the first substantive engineering design was by Farquhar in $1971,{ }^{3}$ and the first major NASA policy assessment was the Report of the National Commission on Space (The Paine Report) in 1986 (Ref. 4).
} 
Orbital Docking System (ODS) becomes in this concept a utility module. This module becomes a structure to host spacecraft systems such as power, cooling, attitude control, while providing a flight-proven airlock and supports an Internal Docking System Standard (IDSS) compatible docking interface. All of this hardware is currently in storage at NASA KSC.

The advocates for these concepts emphasize the value of ISS as a "base camp," with very direct dependence upon the systems, years of experience, and existing international agreements for this facility.

Although this paper is limited to discussion of thinking in the US on long-duration human operations beyond LEO, other space-faring countries, of course, have given some substantive consideration to operating in cis-lunar space. Rather than pursue new technologies, many of the non-US concept studies propose adapting existing hardware and designs, again apparently to save time, money, and to leverage existing experience (cf., the Russian OPSEK Project ${ }^{8}$ ).

\section{TransHab and Beyond: the Future In-Space Operations "Gateway"}

The earliest substantial design for a long-duration habitation system to follow ISS was probably the NASA JSC Transportation Habitation (aka, TransHab), an inflatable system that was pursued to early prototypes in the late 1990s. The TransHab concept had an impressive influence: the basic designs and patents were acquired and developed further by Bigelow Aerospace over the last decade (see below) and the Decade Planning Team/NASA Exploration Team pursued the engineering designs for inflatables further and recommended this future capability to NASA management as well as to the White House Office of Management and Budget. [TransHab/DPT/NExT-derived space habitation systems are used to illustrate the 2004 White House Vision for Space Exploration.]

Over the past half-decade, the Future In-Space Operations (FISO) colleagues have advanced operational concepts beyond those originally proposed by the DPT/NExT for inflatable inspace habitats. They have emphasized the Earth-Moon L1 and L2 sites, both as venues for lunar exploration with robots and humans, for example, as well as for preparation for longerduration human voyages beyond the Earth-Moon system (see

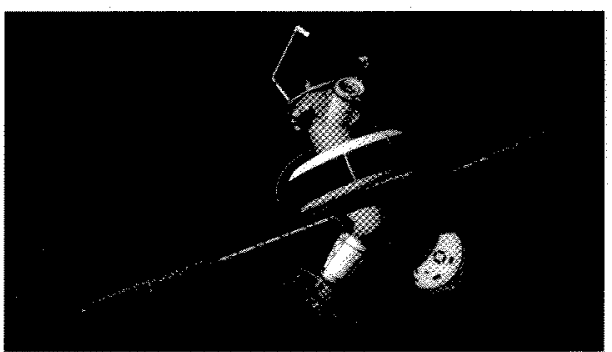

Figure 3: Concept for an inflatable "Gateway" docked with an MPCV and a lunar surface robot. The "Gateway" concept has been proposed as both an operational facility (usually at Earth-Moon L1,2) and a "stepping stone" precursor to subsequent very long-duration human missions. Lester and Thronson in this conference and Refs. 9, 10).

Advocates for these concepts generally emphasize the advantages of inflatable technologies over hard-sided designs, including its apparent extensibility to subsequent more ambitious human voyages, although probably requiring more technology development and time.

\section{The Bigelow Aerospace LLC BA 330}

More than a decade ago, Bigelow Aerospace ${ }^{11}$ (BA) acquired the designs and patents for the NASA TransHab concept and has pursued a series of increasingly sophisticated designs for cis-lunar operation, including a pair of orbiting prototypes (i.e., Genesis $I$ and $I I$ ).

The current plan for BA is to develop the inflatable BA 330 , which as its name suggests, has a usable volume of $330 \mathrm{~m}^{3}$ with the capability of supporting a crew of six in LEO and beyond. Depending upon launch vehicle, the BA 330 may operate throughout cis-lunar space with the capability of docking with other systems and modules to extend its capabilities, especially its volume, in a relatively straightforward manner.

\section{The Imperative of Near-Term Accomplishments in Human Exploration Beyond LEO}

The ISS has been an engineering and management success, especially considering the numerous international partners, and has been continuously occupied for more than a decade. Given the conventional timeline for major space programs of all kinds, it is reasonable - and sobering - to expect that the next major human habitation achievement, presumably beyond LEO, will occur when the ISS is more than a quarter century old.

Political leaders understand that the challenge of major achievements within a short period of time wonderfully concentrates human talent and financial resources. In 2004, President Bush declared a goal for NASA of returning 
to the lunar surface within 16 years. In 2010, President Obama changed the primary goal for America's human space flight program to a mission to a near-Earth asteroid within 15 years. The merits of either goal may be debated, but in both cases very experienced political leaders unambiguously recognized that a major accomplishment within a specific period of time had the desired effect of focusing NASA priorities.

The justifications for a near-term - say, about a decade - major achievement in human space flight are numerous.

\section{A. Next-Generation Launch Vehicles and Near-Future Destinations in Cis-Lunar Space}

A successful long-duration habitat is one of the generally recognized small number of essential capabilities necessary for long-duration human voyages beyond the Earth-Moon system. According to current national planning, the Space Launch System (SLS) and MPCV will be available in the time period that we discuss here, thereby providing deep-space human transporation capability. Developing an affordable cis-lunar habitat in the near term, which must be developed in any case, provides an initial destination for this capability.

Operating with humans beyond LEO for weeks or months at a time will also be an unambiguous political demonstration of a growing capability for longer-duration human missions. This will be an important element in sustaining the funding necessary for even more ambitious subsequent missions. Related to this, it is reasonable to expect that a successful cis-lunar habitation system will have the additional desirable effect of sustaining funding for other key technologies. Advances in in-space propulsion, for example, another critical capability to be mastered for long-duration human space flight, may be more politically sustainable if a cis-lunar habitation system is already operating. The reverse may not, however, be true: space propulsion without a successful habitation program may well be politically problematic. Similarly with other major elements of human exploration beyond LEO: how politically tenable are major entry, descent, and landing (EDL), astronaut EVA capabilities of any kind, or in-situ resource utilization (ISRU) investments without the cis-lunar habitation architecture for astronauts to operate effectively and comfortably? It is for these reasons that we refer to habitation systems beyond LEO as a "lynch pin" for human exploration.

\section{B. Learning by Doing, Sooner Rather than Later}

There is a breadth of development efforts on - or proposed to be on - ISS, as well as using terrestrial facilities, that are relevant to human voyages beyond the immediate vicinity of the Earth-Moon system. This is important work. At the same time, a great deal can be learned no other way than by actual deployment of a habitation system. And the sooner that such a facility is deployed, the more promptly can these lessons be incorporated. And this is certainly the case, even if the facility does not support the longest-duration voyages, although may be extensible or upgradable to such a capability. Indeed, it is almost certain that an essential understanding about how to operate beyond the immediate vicinity of the Earth can only be learned by such a deployment. And the sooner that this is accomplished, the further ahead can be human exploration beyond the Earth-Moon system. This is the philosophy behind the Bigelow Aerospace series of Genesis prototype inflatable habitat systems, which have been in orbit now for some years.

Significant compromises in performance, with concomitant major cost and schedule savings, can be made by developing a habitation system that does not from the start, for example, have the capability for astronaut EVA, offer radiation protection more than that required for a few months of occupation for each crew member, or uses highly efficient closed-cycle life support. It is critical for such capabilities to be developed if humans are to travel beyond the Earth-Moon system, but more limited performance seems worthwhile, if it results in near-term, more affordable experience that can be gained no other way. That is, although identifying the priority activities for humans beyond LEO may not be necessary for the first major "stepping stone" facility discussed here, this will be necessary for substantially more capable habitats.

It seems desirable to design and build in from the start extensibility in an early habitat so that it is straightforward to move from a simple, much more affordable cis-lunar habitat to a system that is capable of more ambitious accomplishments. Nevertheless, waiting for the development of systems required, say, for a mid-2030s mission to Mars, although unnecessary for a mid-2020s cis-lunar demonstration mission, seems to be an expensive, slow, not very instructive, and consequently politically very problematic strategy.

\section{Employ Experienced Talent and Lessons Learned from ISS Design and Deployment}

NASA's space and Earth science programs have learned a lesson that may be relevant to human exploration: compromises may be made and disparate communities will have to be accommodated, but there should always be a series of major programs moving through the pipeline toward flight readiness. Missions of increasing capability, taking advantage of experienced teams, building upon expensive technology investments, and sustaining 
international partnership move forward using discoveries by existing missions often as the core justification for the follow-on programs.

When the ISS nears the end of its operational life - within the professional careers of many readers - the managers, executives, engineers and technologists will quickly retire or transfer to other programs, which is already happening as the ISS ends its period of construction and the Shuttle program is over. That is, experience in engineering design and management of the ISS deployment will soon be lost, unless there is a program underway that can effectively apply the costly, hard-won experience that made the program successful.

Fortunately, there has been extensive design work in the past few years and development investments on ISS, as well as prototype missions such as those by Bigelow Aerospace (above). Thus, NASA may well be able to make a decision in the near future to build a basic habitation system in time for its construction to take advantage of engineers and managers experienced with the development and deployment of ISS of the past several years.

Success with the ISS offers an additional opportunity, as noted in Section IIC above: extensive use of existing ISS hardware that has been designed and, to some degree, already tested for space operations with which current ISS staff is familiar. This may, however, compromise extensibility; that is, the habitat that can be developed quickly and launched within a decade using experienced personnel and components may not be a straightforward "stepping stone" for subsequent systems necessary for longer very long-duration human voyages. Nevertheless, there appear to be options for NASA in a constrained budgetary environment should policymakers conclude that a habitation system must be developed beyond LEO before ISS reaches the end of its operational life and trained personnel are quickly lost.

A near-future habitation system beyond LEO, taking advantage of current ISS personnel, ussing existing hardware, or by reasonable compromise on the early performance of a near-term habitation system, has the immediate knock-on effect of freeing resources for the development of capabilities needed for more ambitious habitats to follow.

\section{Build on Existing ISS National and International Agreements}

No less than the engineering and management excellence of the ISS, coordinating international partners over decades has been at the core of the program's success. Considerable effort has been expended to establish and maintain these international partnerships, agreements are in place, and the senior managers from the contributing countries are familiar with one another. It would seem to be valuable to build upon these agreements for the programs that follow the ISS. Indeed, a superficial examination of the agreements among the ISS partners suggests that an international cis-lunar habitat can be coordinated among partners following closely the model of ISS. This lesson appears to be well-learned by the international partners, who are in the process of developing the "Global Exploration Strategy" for the space agencies as this is being written.

\section{Conclusions}

Several assessments over the past decade have recommended building on the management and engineering success of ISS to develop a follow-on capability for humans to operate comfortably beyond LEO and prepare for very long-duration voyages beyond the Earth-Moon system. Numerous architectures have identified such a facility as one of the few truly enabling capabilities that will bridge the gap between the current capability for successful LEO operations and human exploration deeper into space.

We briefly summarize some concepts for cis-lunar habitats and present justifications for why such a facility should be considered as a priority for near-term deployment (i.e., within a decade). The justifications include (1) taking advantage of the experience gained with the ISS program before it is lost; (2) learning and applying early lessons via actual space operations that cannot be duplicated by engineering design or terrestrial prototypes alone; (3) demonstration of a worthwhile accomplishment that is in the direct path to subsequent achievements in human space flight, including providing a destination for new launch systems being developed over the next several years; (4) building upon already-existing international agreements; and (5), in a constrained financial environment, deploy a less-capable, more-affordable habitat in the near term to free up resources necessary for development of advanced systems required only for the most ambitious human missions.

\section{Acknowledgments}

This work benefitted from discussions with Michael Raftery. 


\section{References}

'Garber, S., and Asner, G. NASA's Decadal Planning Team and the Policy Formulation of the Vision for Space Exploration. URL: http:/history.nasa.gov/DPT/DPT.htm [Cited 1 September 2011 ].

${ }^{2}$ Lester, D. F., and Thronson, H. A., "Human Space Exploration and Human Spaceflight: Latency and The Cognitive Scale of the Universe," Space Policy, Vol. 27, 2011, pp. 89-93,

${ }^{3}$ Farquhar, R., "The Utilization of Halo Orbits in Advanced Lunar Operation," 1971, Goddard Space Flight Center, NASA Technical Note D-6365.

4 "Pioneering the Space Frontier: Report of the National Commission on Space" (The Paine Report), 1986 URL: http://history.nasa.gov/painerep/begin.html [Cited 1 September 2011].

${ }^{5}$ The Deep Space Habitat (DSH) of the NASA Human Architecture Team (HAT), 2011, URL: http://ntrs.nasa.gov/archive/nasa/casi.ntrs.nasa.gov/20110006982 2011003312.pdf [Cited 1 September 2011].

${ }^{6}$ Raftery, M. "Using ISS for Human Exploration Beyond LEO," Future In-Space Operations (FISO) telecon colloquium archives, 2011, URL: http://spirit.as.utexas.edu/ fiso/telecon.htm [Cited 1 September 2011]

${ }^{7}$ Hatfield, C. A., "Using Exissting ISS Hardware to Prepare for Exploration Beyond LEO," Future In-Space Operations (FISO) telecon colloqium archives, 2011, URL: http://spirit.as.utexas.edu/ fiso/telecon.htm [Cited 1 September 2011]

${ }^{8}$ Zak, A., "A Concept of the Russian Successor to the ISS," 2009, URL: http:/www.russianspaceweb.com/opsek.html [Cited 1 September 2011].

${ }^{9}$ Thronson, H. A., and Talay, T., "Gateway' Architectures: A Major 'Flexible Path' Step to the Moon and Mars after the International Space Station?", The Space Review, 2010, URL: http://www.thespacereview.com/article/1561/1 [Cited 1 September 2011].

${ }^{10}$ Thronson, H. A., Lester, D. F., Moe, R., and Sullivan, G., "Review of US Concepts for Post-ISS Space Habitation Facilities and Future Operations," AIAA Space 2010, Washington, D.C., Manuscript 818583, 2010.

${ }^{11}$ Bigelow Aerospace LLC URL: http:/www.bigelowaerospace.com/ [Cited 1 September 2011]. 